\title{
Fano resonance in a normal metal/ferromagnet-quantum dot-superconductor device
}

\author{
Lin Li, ${ }^{1}$ Zhan Cao, ${ }^{2}$ Hong-Gang Luo, ${ }^{2,3, *}$ Fu-Chun Zhang, ${ }^{4,5}$ and Wei-Qiang Chen ${ }^{1, \dagger}$ \\ ${ }^{1}$ Department of Physics, Southern University of Science and Technology of China, Shenzhen 518005, China \\ ${ }^{2}$ Center of Interdisciplinary Studies and Key Laboratory for Magnetism and Magnetic Materials of the Ministry of Education, \\ Lanzhou University, Lanzhou 730000, China \\ ${ }^{3}$ Beijing Computational Science Research Center, Beijing 100084, China \\ ${ }^{4}$ Department of Physics, Zhejiang University, Hangzhou 310027, China \\ ${ }^{5}$ Department of Physics and Center of Theoretical and Computational Physics, The University of Hong Kong, Hong Kong, China
}

(Received 18 September 2015; published 30 November 2015)

\begin{abstract}
We investigate theoretically the Andreev transport through a quantum dot strongly coupled with a normal metal/ferromagnet and a superconductor (N/F-QD-S), in which the interplay between the Kondo resonance and the Andreev bound states (ABSs) has not been clearly clarified yet. Here we show that the interference between the Kondo resonance and the ABSs modifies seriously the line shape of the Kondo resonance, which manifests as a Fano resonance. The ferromagnetic lead with spin polarization induces an effective field, which leads to splitting of both the Kondo resonance and the ABSs. The spin polarization together with the magnetic field applied provides an alternative way to tune the line shape of the Kondo resonances, which is dependent of the relative positions of the Kondo resonance and the ABSs. These results indicate that the interplay between the Kondo resonance and the ABSs can significantly affect the Andreev transport, which could be tested by experiments.
\end{abstract}

DOI: 10.1103/PhysRevB.92.195155

PACS number(s): 74.45.+c, 73.63.Kv, 72.15.Qm, 75.20.Hr

\section{INTRODUCTION}

In the past years, the interplay between the Kondo effect and superconductivity has been intensively investigated in hybrid superconductor nanostructures [1-24]. In a superconductorquantum dot-superconductor (S-QD-S) device, the Josephson current shows an interesting $0-\pi$ phase transition at $T_{K} / \Delta \sim 1$, where $T_{K}$ is the normal state Kondo temperature, and $\Delta$ is the superconducting gap. At the transition point, the energy gained from the formation of the Kondo singlet can exceed the energy gap, which leads to the crossing of Andreev bound states (ABSs) [1-13]. Furthermore, if one electrode of the QD is replaced by a normal or ferromagnetic lead, namely for a normal metal/ferromagnet-quantum dot-superconductor (N/F-QD-S) device, the subgap transport shows much richer features [14,25-27]. For example, the Kondo effect enhancement of Andreev transport has been observed in odd occupation regimes [16,25]. The coexistence of Kondo resonance and $\mathrm{ABSs}$ has been proposed and/or observed in the superconductor-QD devices [26-29]. However, the interplay between the Kondo resonance and the ABSs in such a device has not been explored in detail.

It is well known that in the N/F-QD-S device the Andreev levels induced by the proximity of the superconductor will be broadened, whose width is roughly proportional to the coupling strength with the normal or ferromagnetic lead. At the same time, the coupling with the normal or ferromagnetic lead will also lead to a Kondo resonance due to the screening of the spin in the QD if the system is in the Kondo regime. As mentioned above, both these two subgap features have been observed experimentally [26]. A natural question arises: is there any interplay between these two subgap features? In this work we explore theoretically the interaction between these two distinguished features and find that the line shape

\footnotetext{
*luohg@1zu.edu.cn

†chen.wq@sustc.edu.cn
}

of the Kondo resonance can be modified seriously by the interference between the Kondo resonance and the ABSs, which can be attributed to Fano resonance [30,31].

The experimental observations that the Fano resonance modifies the line shape of the Kondo resonance have been reported about two decades ago [32-35], in which the Kondo resonance has been uncovered by scanning tunneling spectroscopy of a magnetic adatom on metal surface. There the asymmetric line shape of the Kondo resonance observed resulted from the interference between a symmetric Kondo resonance (discrete channel) and the metal surface states (continuum channel) [36]. Furthermore, it was found that in the strongly coupling cases, for example, Ti/Au and Ti/Ag [35,37], the broadening impurity level has a significant contribution to the line shape of the Kondo resonance observed experimentally [38]. These works indicated that the Fano resonance is popular in such a strongly correlated impurity system (for an overview one can refer to Ref. [31]).

In this work we explore the Fano resonance in the N/F-QD-S device. It is shown that the interference between the Kondo resonance and the broadened $\mathrm{ABS}$ s has a significant influence on the Andreev transport. In the N-QD-S case, the Kondo resonance peak developed in Andreev transport gradually evolves into an antiresonance dip structure by increasing the coupling strength between the normal lead and the QD. This can be ascribed to the interference of the Kondo resonance fixed around the Fermi level and the broadened ABSs near the Fermi level. Here the broadened ABSs play a key role in changing the line shape of the Kondo resonance from a peak to a dip structure. In the F-QD-S case, the situation is more interesting. It is known that the spin polarization can induce an effective magnetic field $E_{Z \text { eff }}$ [39-43], which removes the degeneracy of dot level and results in the splitting in Kondo resonance and the ABSs. The interference between the subpeaks of Kondo resonance and split ABSs can lead to fine Fano resonance, as shown schematically in Fig. 1. The spin polarization as well as the magnetic field applied provide a way 


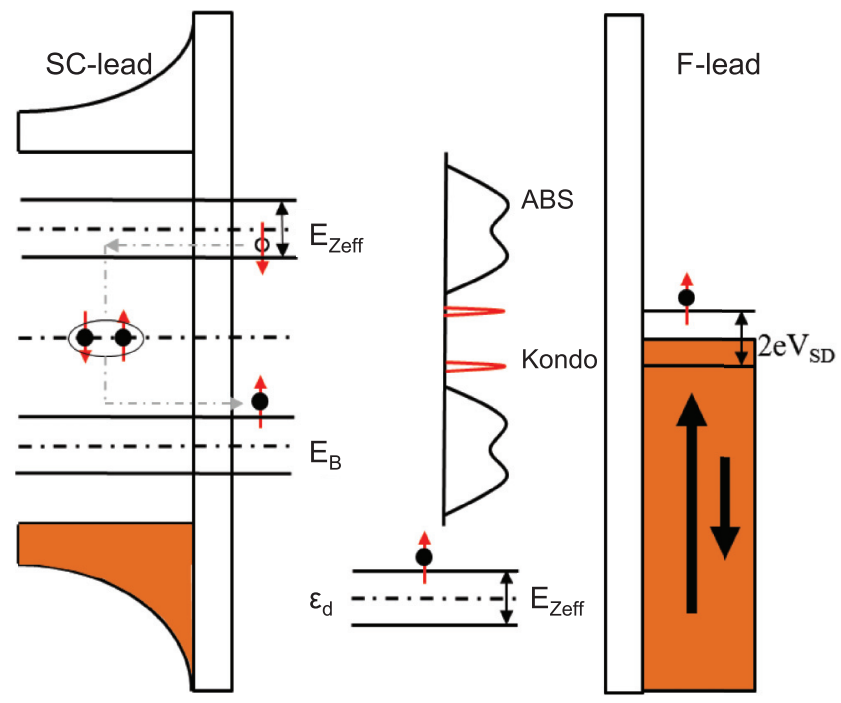

FIG. 1. (Color online) Schematic diagram of the interplay between the Kondo resonance and the Andereev bound states (ABSs) in a F-QD-S device. The spin polarization induces an effective field $E_{\text {Zeff }}$, which removes the degeneracy in dot level $\varepsilon_{d}$ and splits the ABSs and Kondo resonance. When the split ABSs and subpeak of Kondo resonance get close to each other, the interference between them modifies the line shape of the Kondo resonance, which affects the Andreev transport.

to tune the subgap physics of the Andreev transport in such a device, which can be further tested by future experiments.

The paper is organized as follows. In Sec. II we introduce the Anderson impurity model and outline the formalism on the Andreev transport. In Sec. III we discuss the numerical results of Andreev transport in the N/F-QD-S device, and analyze the result obtained by using the Fano resonance picture. Finally, a brief summary is given in Sec. IV.

\section{THE MODEL AND FORMALISM}

The N/F-QD-S device can be described by the Anderson impurity model [44]

$$
H=H_{L}+H_{D}+H_{V},
$$

where

$$
H_{L}=\sum_{k \alpha \sigma} \varepsilon_{k \alpha \sigma} c_{k \alpha \sigma}^{\dagger} c_{k \alpha \sigma}-\Delta \sum_{k \sigma}\left(c_{k S \uparrow}^{\dagger} c_{-k S \downarrow}^{\dagger}+\text { H.c. }\right)
$$

is the Hamiltonian of leads $\alpha=S, N / F$. The second term is only available in the superconducting lead. The operator $c_{k \alpha \sigma}^{\dagger}$ $\left(c_{k \alpha \sigma}\right)$ represents the creation (annihilation) of an electron with the energy $\varepsilon_{k \alpha \sigma}$ in superconductor or normal/ferromagnetic leads, respectively.

$$
H_{D}=\sum_{\sigma} \varepsilon_{d \sigma} d_{\sigma}^{\dagger} d_{\sigma}+\frac{U}{2} \sum_{\sigma} n_{\sigma} n_{\bar{\sigma}}
$$

is the dot Hamiltonian, in which $\varepsilon_{d \sigma}$ is the dot level, $d_{\sigma}^{\dagger}\left(d_{\sigma}\right)$ is the creation (annihilation) operator of electron in QD, $n_{\sigma}=$ $d_{\sigma}^{\dagger} d_{\sigma}$, and $U$ is the Coulomb repulsion.

$$
H_{V}=\sum_{k \alpha \sigma}\left(V_{\alpha} c_{k \alpha \sigma}^{\dagger} d_{\sigma}+\text { H.c. }\right)
$$

is the Hamiltonian describing the coupling between the dot and leads with the tunneling amplitude $V_{\alpha}$. The spin polarization in the ferromagnetic electrode leads to the spin-dependent dotlead coupling $\Gamma_{F \uparrow(\downarrow)}=\Gamma_{F}(1 \pm P) / 2 . \quad \Gamma_{F}=\Gamma_{F \uparrow}+\Gamma_{F \downarrow}=$ $\pi\left|V_{F}\right|^{2} \rho_{F}$ is the coupling strength, and $\rho_{F}=\rho_{F \uparrow}+\rho_{F \downarrow}$ is the density of states of the normal/ferromagnetic lead. $P$ is the spin polarization and $P=0$ denotes a normal electrode. It is known that the ferromagnetic proximity effect induces an effective exchange field on the dot level due to the spin-dependent charge fluctuation [41-43]. This behavior can be treated by the Haldane's scaling theory [45], and the modification on the dot level is

$$
\delta \varepsilon_{d \sigma}=\int \frac{d \varepsilon}{\pi}\left\{\frac{\Gamma_{F \sigma}[1-f(\varepsilon)]}{\varepsilon_{d \sigma}-\varepsilon}+\frac{\Gamma_{F \bar{\sigma}} f(\varepsilon)}{\varepsilon-U-\varepsilon_{d \bar{\sigma}}}\right\} .
$$

The first (second) term denotes charge fluctuation between the single occupation state and empty (double occupation) state on the dot level $[41,42]$.

The subgap transports through the device can be derived by Nambu Green's function

$$
\mathbf{G}_{d \sigma}(\varepsilon)=\left[\begin{array}{cc}
\left\langle\left\langle d_{\sigma} ; d_{\sigma}^{\dagger}\right\rangle\right\rangle_{\varepsilon} & \left\langle\left\langle d_{\sigma} ; d_{\bar{\sigma}}\right\rangle\right\rangle_{\varepsilon} \\
\left\langle\left\langle d_{\bar{\sigma}}^{\dagger} ; d_{\sigma}^{\dagger}\right\rangle\right\rangle_{\varepsilon} & \left\langle\left\langle d_{\bar{\sigma}}^{\dagger} ; d_{\bar{\sigma}}\right\rangle\right\rangle_{\varepsilon}
\end{array}\right] .
$$

All the components in $\mathbf{G}_{d \sigma}(\varepsilon)$ can be calculated by equation of motion approach $[13,14,28,46-50]$. In the frame of HartreeFock mean field, the dot Green's function reads

$$
\mathbf{G}_{d \sigma}^{\mathrm{HF}}(\varepsilon)=\left[\varepsilon \hat{I}-\tilde{\varepsilon}_{d \sigma} \hat{\sigma}_{z}-\hat{\Sigma}^{0}(\varepsilon)-\hat{\Sigma}^{\mathrm{HF}}(\varepsilon)\right]^{-1},
$$

where the noninteracting self-energy $\Sigma_{11}^{0}(\varepsilon)=\Sigma_{22}^{0}(\varepsilon)=$ $-i\left(\Gamma_{F}+\frac{\theta(|\varepsilon|-\Delta)|\varepsilon|}{\sqrt{\varepsilon^{2}-\Delta^{2}}} \Gamma_{S}\right), \quad \Sigma_{12}^{0}(\varepsilon)=\Sigma_{21}^{0}(\varepsilon)=\frac{\Delta}{\sqrt{\Delta^{2}-\varepsilon^{2}}} \Gamma_{S}$, with $\Gamma_{S}=\pi\left|V_{S}\right|^{2} \rho_{S} . \quad \tilde{\varepsilon}_{d \sigma}=\varepsilon_{d \sigma}+\delta \varepsilon_{d \sigma}$ is the renormalized dot level. $\delta \varepsilon_{d \sigma}$ plays a role of effective field $E_{Z \text { eff }}$ on the dot level. $\Sigma^{\mathrm{HF}}=\hat{\sigma}_{z} U\left\langle\hat{n}_{\bar{\sigma}}\right\rangle+\hat{\sigma}_{x} \Delta_{d}$ is the interacting self-energy, where $\Delta_{d}=U\left\langle d_{\sigma}^{\dagger} d_{\bar{\sigma}}^{\dagger}\right\rangle$ is the proximity induced order parameter $[49,51]$.

In order to capture the Kondo physics, the higher-order Green's functions should be taken into account. We truncate the hierarchy of Green's function by Lacroix's scheme [46]. It qualitatively captures the characters of Kondo effect even at zero temperature [52] or particle-hole symmetry [53]. Although the equation of motion approach tends to underestimate the Kondo temperature, it can properly capture the competition between Kondo effect and superconductivity [13]. After some straightforward calculations, the dot Green's function obtained is

$$
\mathbf{G}_{d \sigma}(\varepsilon)=\left(\varepsilon \hat{I}-\tilde{\varepsilon}_{d \sigma} \hat{\sigma}_{z}-\hat{\Sigma}^{0}-\hat{\Sigma}^{K}\right)^{-1},
$$

where $\quad \hat{\Sigma}^{K}=\hat{\sigma}_{z} U\left\langle\hat{n}_{\bar{\sigma}}\right\rangle \frac{\left[\varepsilon-\tilde{\varepsilon}_{d \sigma}-\Sigma_{11}^{0}(\varepsilon)-P_{\sigma}(\varepsilon)\right]+Q_{\sigma}(\varepsilon) /\left\langle\hat{n}_{\bar{\sigma}}\right\rangle}{\varepsilon-\tilde{\varepsilon}_{d \sigma}-\Sigma_{11}^{0}(\varepsilon)-U\left(1-\left\langle\hat{n}_{\bar{\sigma}}\right\rangle\right)-P_{\sigma}(\varepsilon)}+$ $\hat{\sigma}_{x} \Delta_{d} \frac{\Sigma_{12}^{0}(\varepsilon)}{\varepsilon+\tilde{\varepsilon}_{d \bar{\sigma}}-\Sigma_{11}^{0}(\varepsilon)}$ is the self-energy containing Kondo correlation, and the notations $P_{\sigma}$ and $Q_{\sigma}$ read

$$
\begin{aligned}
P_{\sigma}(\varepsilon)= & {\left[\Omega_{1 \sigma}(\varepsilon)+\Omega_{2 \sigma}(\varepsilon)\right]-U\left[A_{1 \sigma}(\varepsilon)-A_{2 \sigma}(\varepsilon)\right], } \\
Q_{\sigma}(\varepsilon)= & \left(\varepsilon-\tilde{\varepsilon}_{d \sigma}-U\left\langle n_{d \bar{\sigma}}\right\rangle\right)\left[A_{1 \sigma}(\varepsilon)-A_{2 \sigma}(\varepsilon)\right]+\left\langle n_{d \bar{\sigma}}\right\rangle \\
& \times\left[\Omega_{1 \sigma}(\varepsilon)+\Omega_{2 \sigma}(\varepsilon)\right]-\left[B_{1 \sigma}(\varepsilon)+B_{2 \sigma}(\varepsilon)\right],
\end{aligned}
$$


where

$$
\begin{gathered}
A_{i \sigma}(\varepsilon) \approx \frac{1}{\pi} \int \frac{\Gamma_{\sigma}\left(\varepsilon^{\prime}\right) f\left(\varepsilon^{\prime}\right) \operatorname{Re}\left[G_{\sigma}\left(\varepsilon^{\prime}\right)\right]}{\varepsilon-\varepsilon_{i \sigma}} d \varepsilon^{\prime}, \\
B_{i \sigma}(\varepsilon) \approx \frac{1}{\pi} \int \frac{\Gamma_{\sigma}\left(\varepsilon^{\prime}\right) f\left(\varepsilon^{\prime}\right)}{\varepsilon-\varepsilon_{i \sigma}} d \varepsilon^{\prime}, \\
\Omega_{i \sigma}(\varepsilon)=\frac{1}{\pi} \int \frac{\Gamma_{\sigma}\left(\varepsilon^{\prime}\right)}{\varepsilon-\varepsilon_{i \sigma}} d \varepsilon^{\prime} .
\end{gathered}
$$

In the above expressions $\Gamma_{\sigma}(\varepsilon)=\Gamma_{F \sigma}+\Gamma_{S} \frac{|\varepsilon| \theta(|\varepsilon|-\Delta)}{2 \sqrt{\varepsilon^{2}-\Delta^{2}}}, \varepsilon_{1 \sigma}=$ $\tilde{\varepsilon}_{d \sigma}+\varepsilon^{\prime}-\tilde{\varepsilon}_{d \bar{\sigma}}$, and $\varepsilon_{2 \sigma}=-\varepsilon^{\prime}+2 \tilde{\varepsilon}_{d \bar{\sigma}}+U, i=1,2 . f(\varepsilon)$ is the Fermi distribution function. We can calculate the Green's functions self-consistently and the current through the quantum dot by using the above formulas.

In this work we study the Andreev transport through the N/F-QD-S device, in which an electron injects from a normal/ferromagnetic lead to a superconductor through the quantum dot and a hole with opposite spin reflects the normal/ferromagnetic lead, then a Cooper pair is created in the superconductor lead, and vice versa [28,29,48,50]. Therefore, the Andreev current reads

$$
\begin{aligned}
I_{A}(\varepsilon)= & \frac{2 e}{h} \sum_{\sigma} \int d \varepsilon \Gamma_{F \sigma} \Gamma_{F \bar{\sigma}}\left|G_{d \sigma}^{r}(\varepsilon)_{12}\right|^{2} \\
& \times\left[f_{F}\left(\varepsilon-e V_{s d}\right)-f_{F}\left(\varepsilon+e V_{s d}\right)\right],
\end{aligned}
$$

where $f_{F}\left(\varepsilon \pm e V_{s d}\right)$ is the distribution function of electrons in a normal metal/ferromagnetic lead, and $V_{s d}$ is the bias applied.

\section{NUMERICAL RESULT AND DISCUSSION}

In this section we discuss the numerical results of Andreev transport in the N/F-QD-S device. In odd occupation regimes, the Kondo resonance appears around the Fermi level of N/F lead due to the screening of local moment. Here we pay attention to the interplay between the Kondo resonance and the ABSs, especially the interference between them. In the calculations we assume that the density of states $\rho_{\alpha}=1 / 2 D$ and the temperature $T=0, D$ is the half-band-width of the N/F lead. In addition, we neglect the influence of applied magnetic field on the leads and set $U \rightarrow \infty$ for simplification. In the preset work we take $\Delta$ as the units of energy.

In Fig. 2 we show the Andreev transport in the N-QD-S device, namely the polarization $P=0$. When the quantum dot weakly coupled with normal lead $\left(\Gamma_{N} \ll \Gamma_{S}, \varepsilon_{d}\right)$, the Kondo resonance peak cannot be observed in the conductance as shown in Fig. 2(a). In this case, the position of ABSs level $E_{B}$ is determined by the coupling $\Gamma_{S}$. For $T_{K} \ll \Delta$, the level $E_{B}$ situates away from the Fermi level. In the opposite limit, namely $T_{K} \gg \Delta$, the bound states would be located above the Fermi level. The Andreev level $E_{B}$ goes cross the Fermi level about $\Gamma_{S}=8 \Delta$ and $T_{K} / \Delta \sim 1$, which corresponds to the quantum phase transition between magnetic doublet and screened Kondo singlet ground state $[6,12,13,54]$. The width of the ABSs, which associates with its lifetime, only depends on the coupling $\Gamma_{N}$. In Fig. 2(b) we fix the coupling $\Gamma_{S}=5 \Delta$ and check the evolution of the Kondo resonance with increasing $\Gamma_{N}$. The Kondo resonance peak develops at the Fermi level by increasing the coupling $\Gamma_{N}=\Delta, 1.5 \Delta$. Further increasing $\Gamma_{N}$, the resonance peak evolves into a dip structure, as shown

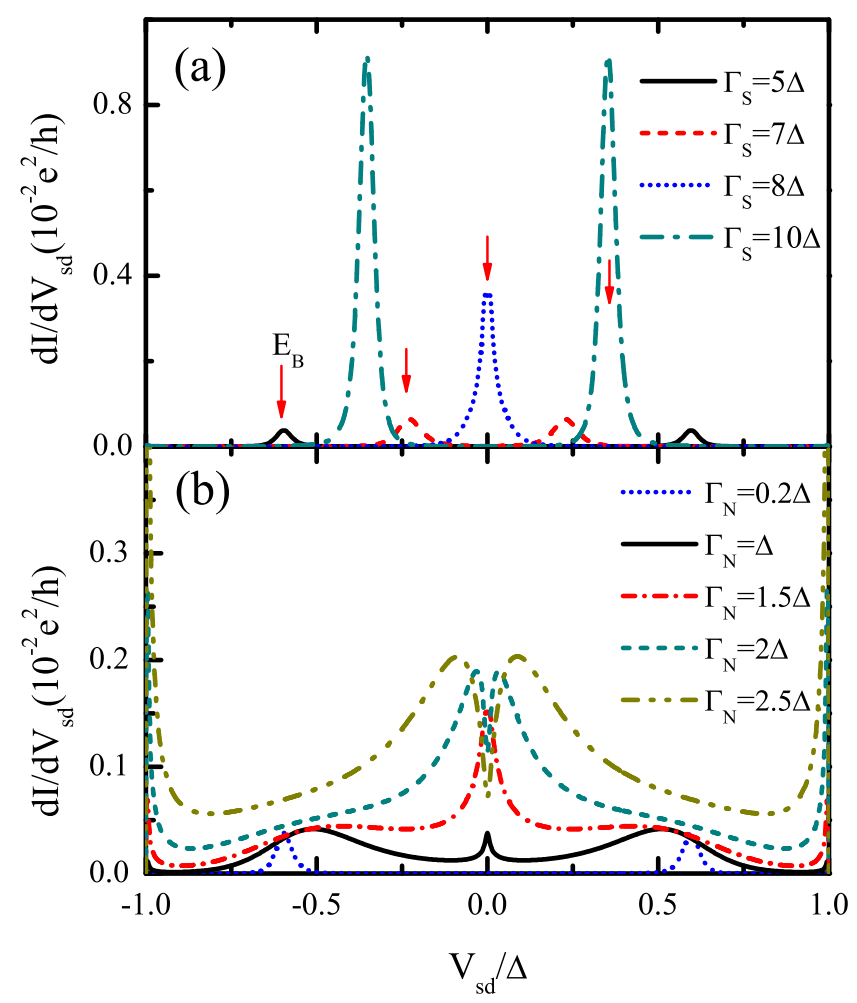

FIG. 2. (Color online) (a) The ABSs dominated by the competition between Kondo effect and superconductivity in the N-QD-S device with the coupling $\Gamma_{N}=0.2 \Delta$ and $\Gamma_{S}=(5,7,8,10) \Delta$. (b) The coexistence of Kondo resonance and ABSs with the coupling $\Gamma_{S}=5 \Delta$ and $\Gamma_{N}=(0.2,1,1.5,2,2.5) \Delta$. Other parameters used: the dot level $\varepsilon_{d}=-5 \Delta$, the temperature $T=0$, the magnetic field $E_{Z}=0$, and the half-band-width $D=20 \Delta$.

for $\Gamma_{N}=2 \Delta, 2.5 \Delta$. This change is ascribed to the interference between the Kondo resonance and the significantly broadened ABSs, as discussed later.

When an external magnetic field is applied and/or the spin polarization in the ferromagnetic lead is present, the Andreev transport displays some interesting splitting behaviors. In particular, the spin polarization induces a local effective field which removes the spin degeneracy in Kondo effect and ABSs [27]. In Figs. 3(a) and 3(b) we show the splitting of the ABSs in the presence of Zeeman energy $E_{Z}$ induced by external magnetic field and spin-polarization $P$, respectively. Differently from the splitting induced by external magnetic field [see Fig. 3(a)], the ferromagnetic polarization induces an imbalance in the local density of states (LDOS) of spin-up and spin-down electrons, as shown in the inset of Fig. 3(b). Due to weak coupling with the $\operatorname{dot} \Gamma_{N}=0.2 \Delta$, the Kondo resonance at the Fermi level does not occur. However, when the quantum dot is strongly coupled with a normal or ferromagnetic lead, the Kondo resonance occurs at the Fermi level, as shown in Fig. 4 (marked by black lines). This Kondo resonance peak becomes split when an external magnetic field [see Fig. 4(a)] or a ferromagnetic lead [see Fig. 4(b)] is applied. Besides the occurrence of the Kondo resonance, another distinguished effect lead by strongly coupling $\Gamma_{N}=1.5 \Delta$ is the broadening of the ABSs, as shown as a broad peak marked by the black lines in Fig. 4(b), which are also split under the external 


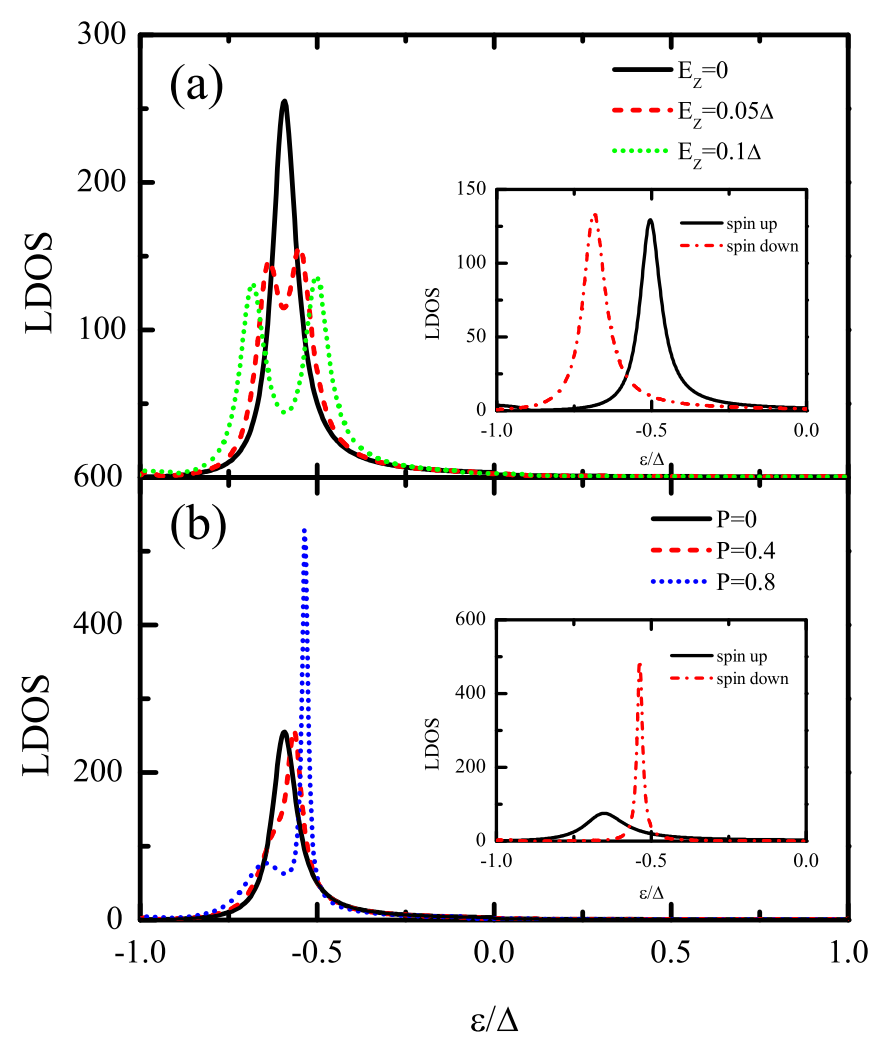

FIG. 3. (Color online) The splitting of the ABSs. (a) The case with different external magnetic field $E_{Z}=0,0.05 \Delta, 0.1 \Delta$ and $P=$ 0 . The inset is the spin-dependent local density of states (LDOS) with $E_{Z}=0.1 \Delta$. (b) The case with different spin polarization with $P=0,0.4$, and 0.8 and $E_{Z}=0$. The inset shows the spin-dependent LDOS with $P=0.8$. Other parameters used are $\varepsilon_{d}=-5 \Delta, \Gamma_{S}=$ $5 \Delta, \Gamma_{N}=0.2 \Delta$, and $D=20 \Delta$.

magnetic field or the ferromagnetic field. The broadening and splitting of ABSs are found to have a significant influence on the line shape of the Kondo resonance. For example, in Fig. 4(b), for $P=0.2 \Delta, 0.4 \Delta$, up to $0.6 \Delta$ the line shape of the split Kondo resonance peaks is obviously different. For the subpeak above the Fermi level, the line shape is a resonance peak, which is similar to that of $P=0$. On the contrary, the line shape of the subpeak below the Fermi level is quite asymmetric [as presented as a red line in Fig. 5(a)], showing a dip-peak structure, which is reminiscent of the Fano resonance. In the following we argue that the broadened ABSs provides a significant continuum which leads to the asymmetric Kondo subpeak by interference effect.

It is well known that the subgap features in the present case involve at most two entities: one is the ABSs and the other is the Kondo resonance if the conditions are met. Thus, the dot Green's function in the subgap regime can be written by introducing $T$ matrix as

$$
G_{d}(\varepsilon)=G_{d}^{0}(\varepsilon)+G_{d}^{0}(\varepsilon) T_{d}(\varepsilon) G_{d}^{0}(\varepsilon),
$$

where $G_{d}^{0}(\varepsilon)$ is the dot Green's function in the subgap, which is nothing but the ABS described approximately by a simple Lorentzian resonance

$$
G_{d}^{0}(\varepsilon)=\frac{Z_{B}}{\varepsilon-E_{B}+i \Gamma_{B}},
$$

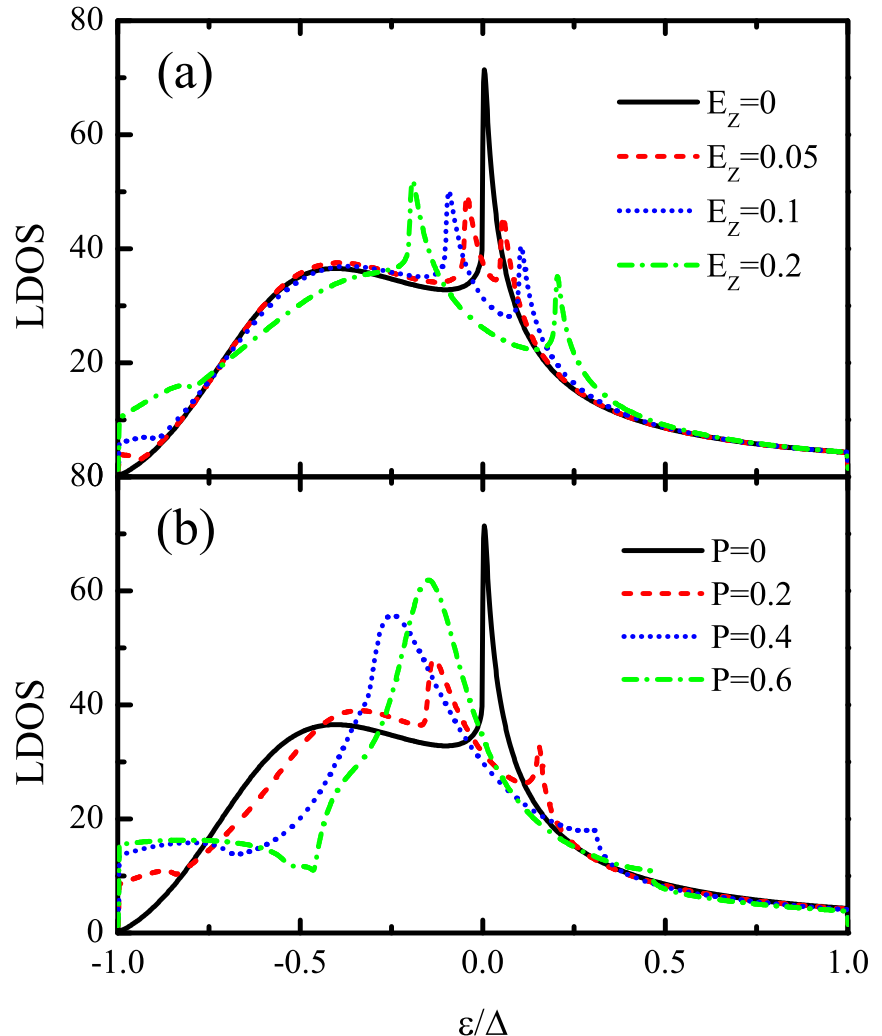

FIG. 4. (Color online) The splitting of the Kondo resonance and the ABSs. (a) The case for different external magnetic field $E_{Z}=$ $0,0.05 \Delta, 0.1 \Delta$ and $P=0$. (b) The case for different spin polarization with $P=0,0.2,0.4$, and 0.6 and $E_{Z}=0$. Other parameters used are the same as those in Fig. 3 except $\Gamma_{N}=1.5 \Delta$.

where $E_{B}, \Gamma_{B}$, and $Z_{B}$ denote the position, the width, and the weight of the ABSs. It is known that $\Gamma_{B} \propto \Gamma_{N / F}$. The $T$-matrix $T_{d}(\varepsilon)$ is mainly composed by Kondo resonance in the Kondo regime

$$
T_{d}(\varepsilon) \approx \frac{\Gamma_{K}}{\pi \rho_{d}^{0}(\varepsilon)} \frac{1}{\varepsilon-\varepsilon_{K}+i \Gamma_{K}},
$$

which is also approximately described by a Lorentzian line shape [38]. $\Gamma_{K}=T_{K}$ is the half-width of Kondo resonance, $\varepsilon_{K}$ is its position.

From Eqs. (15)-(17) the local density of states (LDOS) of the dot in the subgap $\rho_{d}(\varepsilon)=-\frac{1}{\pi} \operatorname{Im} G_{d}(\varepsilon)$ reads

$$
\begin{aligned}
\rho_{d}(\varepsilon)= & -\frac{1}{\pi} \operatorname{Im}\left[G_{d}(\varepsilon)\right] \\
= & \rho_{d}^{0}(\varepsilon)-\pi\left[\rho_{d}^{0}(\varepsilon)\right]^{2} \\
& \times\left\{\left[q_{d}^{2}(\varepsilon)-1\right] \operatorname{Im} T_{d}(\varepsilon)-2 q_{d}(\varepsilon) \operatorname{Re} T_{d}(\varepsilon)\right\},
\end{aligned}
$$

where $\quad \rho_{d}^{0}(\varepsilon)=-\frac{1}{\pi} \operatorname{Im}\left[G_{d}^{0}(\varepsilon)\right] \quad$ and $\quad q_{d}(\varepsilon)=$ $-\operatorname{Re} G_{d}^{0}(\varepsilon) / \operatorname{Im} G_{d}^{0}(\varepsilon)$ is the asymmetry factor. Substituting $T_{d}(\varepsilon)$ into Eq. (18), one can obtain a Fano-like formula for the LDOS in the subgap regime

$$
\rho_{d}(\varepsilon)=\rho_{d}^{0}(\varepsilon) \frac{\left[x+q_{d}(\varepsilon)\right]^{2}}{x^{2}+1},
$$



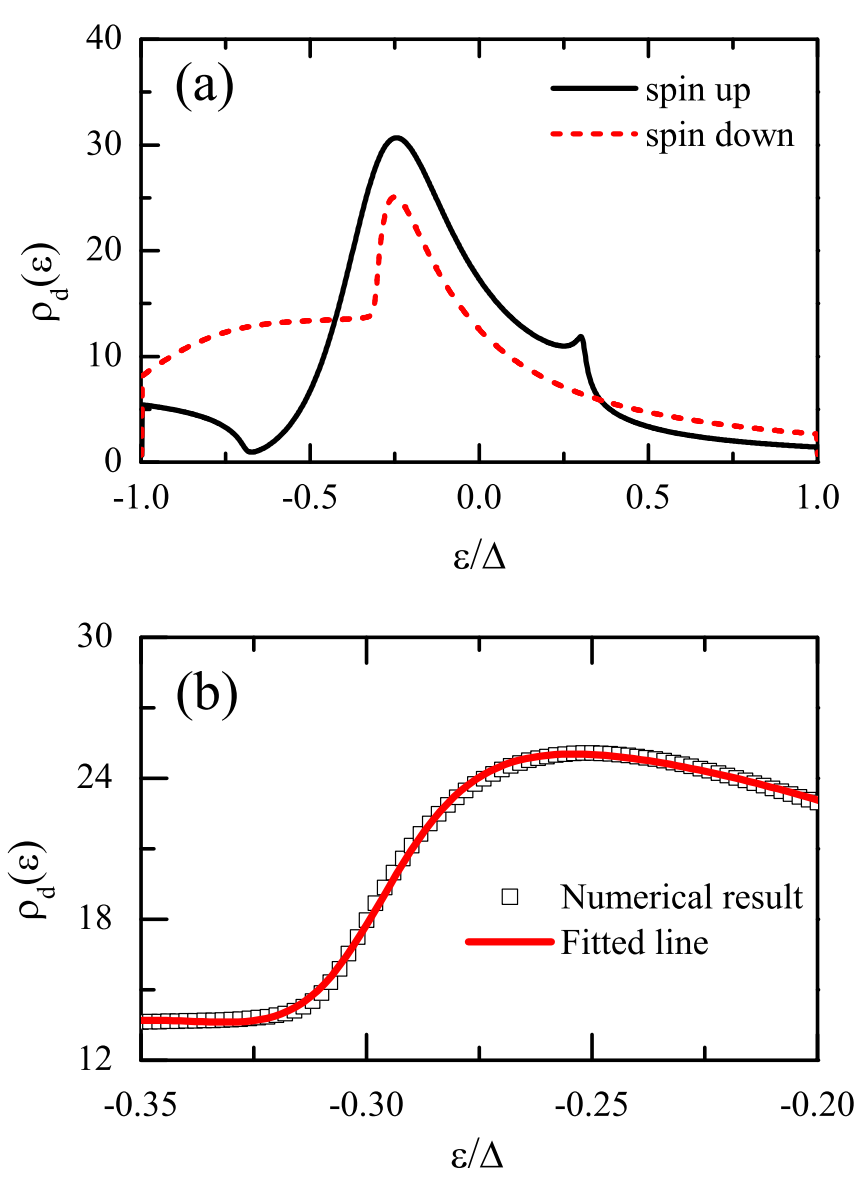

FIG. 5. (Color online) (a) The spin-dependent LDOS with $P=$ 0.4 and $E_{Z}=0$ as given by the dotted line in Fig. 4(b). (b) The asymmetric structure in the LDOS of spin-down electrons is fitted with the theory of Fano resonance.

with $x=\left(\varepsilon-\varepsilon_{K}\right) / \Gamma_{K}$, as given in Ref. [38]. The density of states $\rho_{d}^{0}(\varepsilon)$ can be obtained from Eq. (16),

$$
\rho_{d}^{0}(\varepsilon)=\frac{1}{\pi} \frac{Z_{B} \Gamma_{B}}{\left(\varepsilon-E_{B}\right)^{2}+\Gamma_{B}^{2}} .
$$

Equation (19) can simply capture the in-gap LDOS and the characteristics in Andreev transport, which can be recognized as the coherent superposition of ABSs and Kondo resonance.

In the absence of Kondo resonance $\left(\Gamma_{N} \ll\left|\varepsilon_{d \sigma}\right|\right)$, the ABSs always keep the Lorentzian line shape, even the factor $\left|q_{d}\right| \rightarrow$ $\infty$. By increasing the coupling $\Gamma_{N}$, the Kondo resonance peak appears and evolves into a Fano-dip structure for $\Gamma_{N} \sim\left|\varepsilon_{d \sigma}\right|$ as shown in Fig. 2(b). In this case, the interference between the Kondo resonance and the significant broadened ABSs distorts the line shape of Kondo resonance. In the presence of magnetic field or spin polarization, the interference between the subpeak of Kondo resonance and split ABSs also leads to Fano asymmetric structures as shown in Fig. 4. In Fig. 5(a) we plot the spin-dependent LDOS with $P=0.4$. The Zeeman splitting of the square-root singularity situates symmetrically around the energy gap. This phenomenon has been reported in the previous experimental and theoretical investigations [29,55]. Interestingly, the Kondo resonance splits into a resonant subpeak and a Fano-type asymmetric structure for spin-up

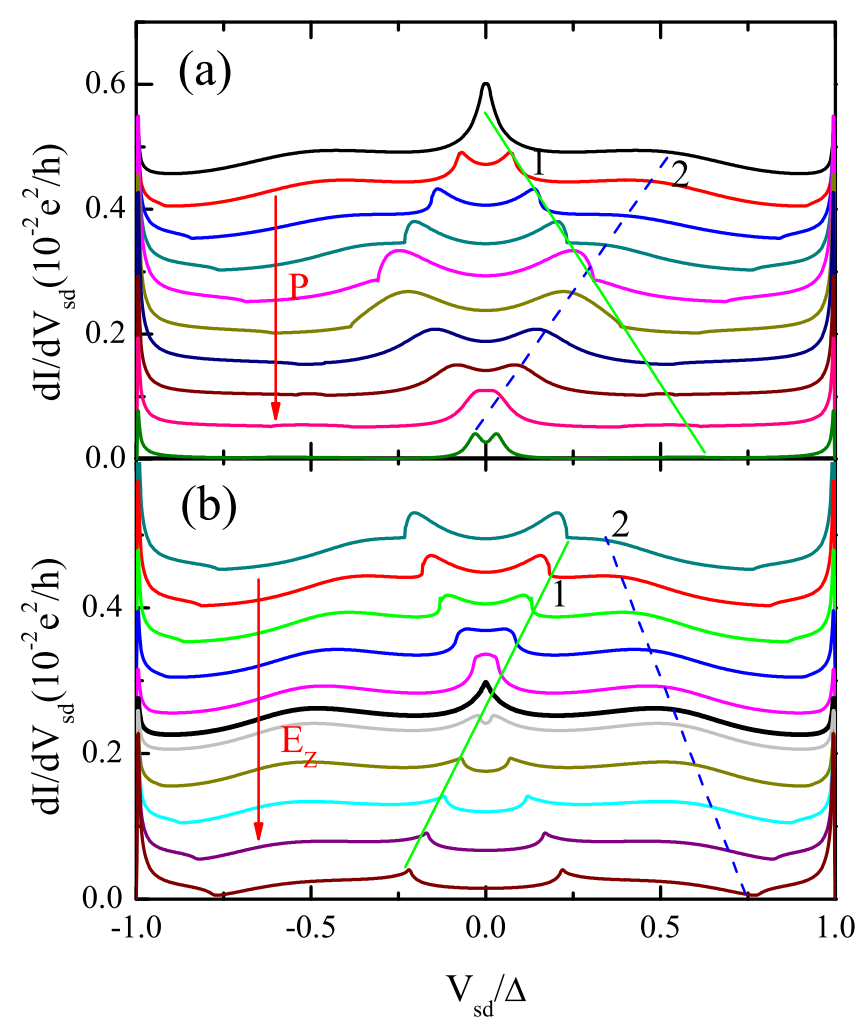

FIG. 6. (Color online) (a) The evolution of ABSs and the splitting of the Kondo resonance due to the spin polarization of lead, the polarization $P$ increases from $P=0$ (the top) to $P=0.9$ (the bottom) with the step 0.1 , and $E_{Z}=0$. (b) The compensation effect of the ABSs and the Kondo splitting by external magnetic fields with $P=0.3$. The Zeeman energy varying from the top $E_{Z}=0$ to the bottom $E_{Z}=0.225 \Delta$ with a step $0.025 \Delta$. The compensation field is $E_{Z}^{c}=0.135 \Delta$, as shown by the bold dark curve. Other parameters used: the dot level $\varepsilon_{d}=-5 \Delta$, the coupling $\Gamma_{S}=5 \Delta$ and $\Gamma_{N}=1.5 \Delta$, and the half-band-width $D=20 \Delta$.

and spin-down electrons, respectively. In Fig. 5(b) we fit the asymmetric structure in the LDOS of spin-down electrons by the qualitative theory of Fano resonance [see Eq. (19)] by using the following parameters: $q_{d}=1.15, T_{K}=0.031 \Delta, E_{B}=$ $-0.2 \Delta, \varepsilon_{K}=-0.3 \Delta, Z_{B}=0.04$, and $\Gamma_{B}=0.12 \Delta$, and the

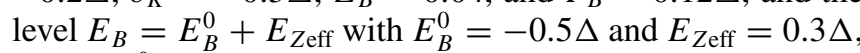
where $E_{B}^{0}$ is the bare level of $\mathrm{ABS}$ in the absence of spin polarization, and $E_{\text {Zeff }}$ is the Zeeman energy induced by the effective field.

In Figs. 6 and 7 we show the evolution of Kondo resonance peak $\left(\Gamma_{N}=1.5 \Delta\right)$ and Fano-dip $\left(\Gamma_{N}=2 \Delta\right)$ structures in Andreev transport spectrum of the F-QD-S device. When the ferromagnetic lead is weakly coupled with quantum dot, the spin-polarization induced effective field $E_{Z \text { eff }}$ is too small to be observed in ABSs. Therefore, we discuss the case that the dot is strongly coupled both with ferromagnetic and superconductor leads. From Fig. 6(a) we can see that the ABSs are split and suppressed by spin polarization as indicated by the blue dashed line 2 . In addition, the Kondo resonance is also split into two side peaks by the induced effective field [39-43]. By increasing $P$ the line shape of the side peaks evolves from a peak to a peak dip, and even to a dip structure. Eventually the Kondo effect is 


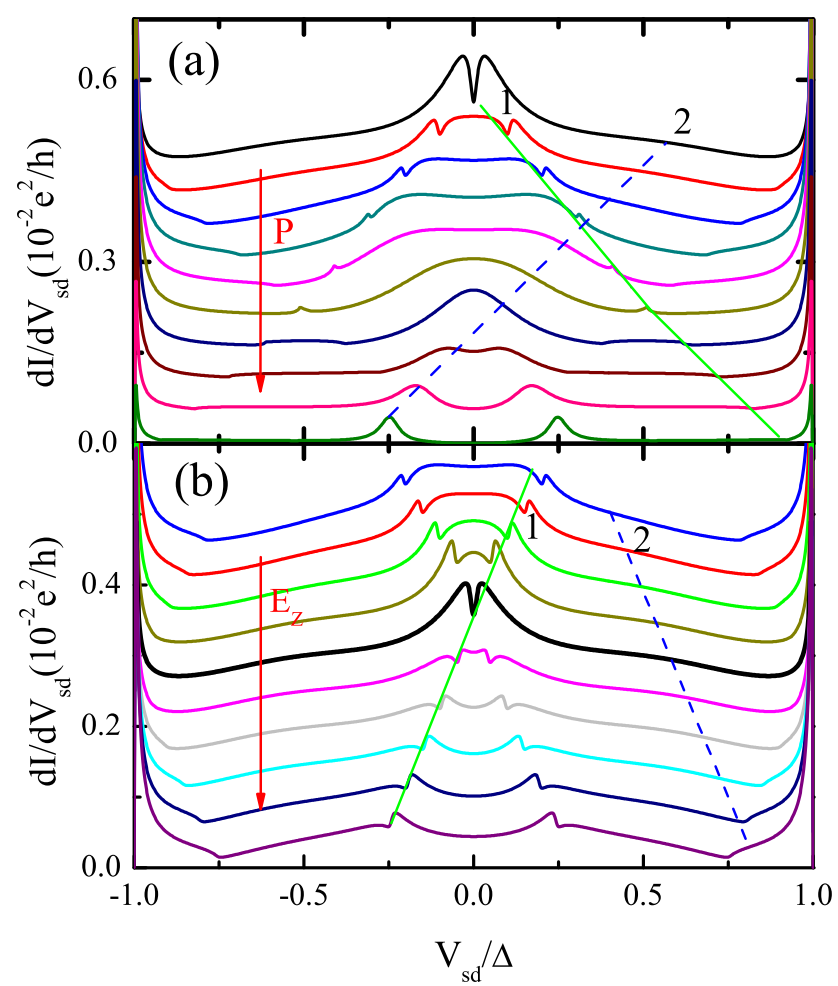

FIG. 7. (Color online) The suppression of ABS and the splitting of Kondo dip due to the spin polarization and its compensation effect by external magnetic field. (a) The polarization $P$ increases from $P=0$ (the top) to $P=0.9$ (the bottom) with the step 0.1 and the Zeeman energy $E_{Z}=0$. (b) The compensation of ABS and the Kondo splitting by external magnetic field varying from the top $E_{Z}=0$ to the bottom $E_{Z}=0.225 \Delta$ with the step $0.025 \Delta$. For the polarization $P=0.2$, the compensation field is $E_{Z}^{c}=0.1 \Delta$, as shown by the bold dark curve. Other parameters used: the dot level $\varepsilon_{d}=-5 \Delta$, the coupling $\Gamma_{S}=5 \Delta$ and $\Gamma_{N}=2 \Delta$, and the half-band-width $D=20 \Delta$.

completely suppressed by the large polarization $P$, as indicated by the green dotted line 1. As shown above, the Fano resonance originates from the interference between the side peaks of Kondo resonance and the split ABSs. At the bias $V_{s d}= \pm \Delta$ the Kondo resonance at the Fermi level of ferromagnetic lead also significantly enhances the Andreev transport. In Fig. 6(b) we show the compensation effect in Andreev transport, since external applied field can counteract the influence of effective field on electron spin. All the split structures in ABSs can be compensated by applied magnetic field. For the polarization $P=0.3$, the corresponding compensated Zeeman energy is $E_{Z}^{c}=0.135 \Delta$ as shown by the bold dark curve in Fig. 6(b). By increasing the external magnetic field, the asymmetric structures first merge into a Kondo resonance peak. For $E_{Z}>$
$E_{Z}^{c}$ the Kondo resonance peak splits again into two subpeaks, which become more and more symmetric, because the Fano interference is suppressed by increasing the distance between the Kondo resonance and ABSs through the Zeeman splitting $E_{Z}$.

In Fig. 7(a) we show the influence of an effective field on antiresonance dip structure in Andreev transport. In the absence of polarization, the antiresonance dip originates from the interference between Kondo resonance and ABSs. In the presence of polarization, the interference between the subpeaks of Kondo resonance and split ABSs can be tuned by increasing the distance between them. As indicated by the green solid line 1, the resonance dip splits into two side-dip structures, which evolve into resonance peaks and then are completely suppressed by increasing the polarization $P$. The ABSs are also split by the effective field and shows crossing behavior. In Fig. 7(b) we show the splitting of ABSs and Kondo resonance can be compensated by applied magnetic field. For the polarization $P=0.2$, the splitting in ABSs can be compensated by the applied magnetic field with $E_{Z}^{c}=0.1 \Delta$. The antiresonance dips display crossing behavior and evolve into asymmetric structures by increasing the magnetic field. In Figs. 6(b) and 7(b) the Fano asymmetric structures at finite bias are pronounced when the split ABSs gets close to the subpeak of Kondo resonance. The line shape is determined by the Fano factor $q_{d}$. Oppositely, the Kondo resonance recovers the peak structure when it is away from the ABSs. Because the dot level can not be significantly broadened by applied magnetic field, the Fano effect can only attribute to the interference between the ABSs and subpeak of Kondo resonance.

\section{SUMMARY}

In conclusion, we have studied the Fano resonance in Andreev transport through the N/F-QD-S device. In N-QD-S device, the interference between the Kondo effect and the broadened ABSs leads to an antiresonance structure at zero bias in Andreev transport. In the presence of spin polarization, the ferromagnetic lead induces an effective magnetic field, which splits the Kondo resonance and ABSs. All the split structures can be compensated by an external applied magnetic field. When the subpeaks of Kondo resonance and split ABSs get close to each other, the interference between them results in Fano-type asymmetric structures at finite bias. The Fano resonance in Andreev transport is expected to be observed in future experiments.

\section{ACKNOWLEDGMENTS}

L.L. gratefully acknowledges useful discussions with Dr. Hua Chen. This work is supported by NSFC (Grants No. 11174115, No. 11325417, No. 11204186, and No. 11274269), PCSIRT (Grant No. IRT1251) of China, and Guangdong Natural Science Foundation 2014A030310137.
[1] A. V. Rozhkov and D. P. Arovas, Phys. Rev. Lett. 82, 2788 (1999).

[2] A. A. Clerk and V. Ambegaokar, Phys. Rev. B 61, 9109 (2000).
[3] M.-S. Choi, M. Lee, K. Kang, and W. Belzig, Phys. Rev. B 70, 020502(R) (2004).

[4] F. Siano and R. Egger, Phys. Rev. Lett. 93, 047002 (2004). 
[5] J. S. Lim and M.-S. Choi, J. Phys. Condens. Matter 20, 415225 (2008).

[6] G. Sellier, T. Kopp, J. Kroha, and Y. S. Barash, Phys. Rev. B 72, 174502 (2005).

[7] J.-P. Cleuziou, W. Wernsdorfer, V. Bouchiat, T. Ondarcuhu, and M. Monthioux, Nat. Nanotechnol. 1, 53 (2006).

[8] C. Buizert, A. Oiwa, K. Shibata, K. Hirakawa, and S. Tarucha, Phys. Rev. Lett. 99, 136806 (2007).

[9] C. Karrasch and V. Meden, Phys. Rev. B 79, 045110 (2009).

[10] D. J. Luitz, F. F. Assaad, T. Novotny, C. Karrasch, and V. Meden, Phys. Rev. Lett. 108, 227001 (2012).

[11] J.-D. Pillet, P. Joyez, R. Zitko, and M. F. Goffman, Phys. Rev. B 88, 045101 (2013).

[12] B.-K. Kim, Y.-H. Ahn, J.-J. Kim, M.-S. Choi, M.-H. Bae, K. Kang, J. S. Lim, R. Lopez, and N. Kim, Phys. Rev. Lett. 110, 076803 (2013).

[13] L. Li, B. B. Zheng, W. Q. Chen, H. Chen, H.-G. Luo, and F. C. Zhang, Phys. Rev. B 89, 245135 (2014).

[14] Q.-F. Sun, H. Guo, and T. H. Lin, Phys. Rev. Lett. 87, 176601 (2001).

[15] A. V. Rozhkov and D. P. Arovas, Phys. Rev. B 62, 6687 (2000).

[16] M. R. Buitelaar, T. Nussbaumer, and C. Schönenberger, Phys. Rev. Lett. 89, 256801 (2002).

[17] M. R. Buitelaar, W. Belzig, T. Nussbaumer, B. Babic, C. Bruder, and C. Schönenberger, Phys. Rev. Lett. 91, 057005 (2003).

[18] M. R. Graber, T. Nussbaumer, W. Belzig, and C. Schönenberger, Nanotechnology 15, S479 (2004).

[19] T. Meng, S. Florens, and P. Simon, Phys. Rev. B 79, 224521 (2009).

[20] V. Koerting, B. M. Andersen, K. Flensberg, and J. Paaske, Phys. Rev. B 82, 245108 (2010).

[21] R. Zitko, M. Lee, R. López, R. Aguado, and M.-S. Choi, Phys. Rev. Lett. 105, 116803 (2010).

[22] A. Martín-Rodero and A. Levy Yeyati, Adv. Phys. 60, 899 (2011).

[23] Eduardo J. H. Lee, X. Jiang, R. Aguado, G. Katsaros, C. M. Lieber, and S. De Franceschi, Phys. Rev. Lett. 109, 186802 (2012).

[24] A. Kumar, M. Gaim, D. Steininger, A. Levy Yeyati, A. MartínRodero, A. K. Huttel, and C. Strunk, Phys. Rev. B 89, 075428 (2014).

[25] A. Eichler, M. Weiss, S. Oberholzer, C. Schönenberger, A. Levy Yeyati, J. C. Cuevas, A. Martín-Rodero, Phys. Rev. Lett. 99, 126602 (2007); A. Eichler, M. Weiss, S. Oberholzer, C. Schonenberger, A. Levy Yeyati, J. C. Cuevas, A. Martin-Rodero, Phys. Rev. B 79, 161407(R) (2009).

[26] R. S. Deacon, Y. Tanaka, A. Oiwa, R. Sakano, K. Yoshida, K. Shibata, K. Hirakawa, and S. Tarucha, Phys. Rev. Lett. 104, 076805 (2010); Phys. Rev. B 81, 121308 (2010).

[27] L. Hofstetter, A. Geresdi, M. Aagesen, J. Nygård, C. Schönenberger, and S. Csonka, Phys. Rev. Lett. 104, 246804 (2010).
[28] M. Krawiec and K. I. Wysokiński, Supercond. Sci. Technol. 17, 103 (2004).

[29] T. Domanski, A. Donabidowicz, and K. I. Wysokinski, Phys. Rev. B 76, 104514 (2007).

[30] U. Fano, Phys. Rev. 124, 1866 (1961).

[31] A. E. Miroshnichenko, S. Flach, and Y. S. Kivshar, Rev. Mod. Phys. 82, 2257 (2010).

[32] Jintao Li, W.-D. Schneider, R. Berndt, and B. Delley, Phys. Rev. Lett. 80, 2893 (1998).

[33] V. Madhavan, W. Chen, T. Jamneala, M. F. Crommie, and N. S. Wingreen, Science 280, 567 (1998).

[34] H. C. Manoharan, C. P. Lutz, and D. M. Eigler, Nature (London) 403, 512 (2000).

[35] T. Jamneala, V. Madhavan, W. Chen, and M. F. Crommie, Phys. Rev. B 61, 9990 (2000).

[36] O. Ujsaghy, J. Kroha, L. Szunyogh, and A. Zawadowski, Phys. Rev. Lett. 85, 2557 (2000).

[37] K. Nagaoka, T. Jamneala, M. Grobis, and M. F. Crommie, Phys. Rev. Lett. 88, 077205 (2002).

[38] H. G. Luo, T. Xiang, X. Q. Wang, Z. B. Su, and L. Yu, Phys. Rev. Lett. 92, 256602 (2004).

[39] J. Martinek, Y. Utsumi, H. Imamura, J. Barnaś, S. Maekawa, J. König, and G. Schön, Phys. Rev. Lett. 91, 127203 (2003); J. Martinek, Y. Utsumi, H. Imamura, J. Barnas, S. Maekawa, J. Konig, and G. Schon, 91, 247202 (2003).

[40] M. S. Choi, D. Sanchez, and R. Lopez, Phys. Rev. Lett. 92, 056601 (2004).

[41] R. Swirkowicz, M. Wilczynski, and J. Barnas, J. Phys. Condens. Matter 18, 2291 (2006).

[42] M. Sindel, L. Borda, J. Martinek, R. Bulla, J. Konig, G. Schon, S. Maekawa, and J. von Delft, Phys. Rev. B 76, 045321 (2007).

[43] L. Li, Y.-Y. Ni, T.-F. Fang, and H.-G. Luo, Phys. Rev. B 84, 235405 (2011).

[44] P. W. Anderson, Phys. Rev. 124, 41 (1961).

[45] F. D. M. Haldane, Phys. Rev. Lett. 40, 416 (1978).

[46] C. Lacroix, J. Phys. F 11, 2389 (1981).

[47] H.-G. Luo, Z.-J. Ying, and S.-J. Wang, Phys. Rev. B 59, 9710 (1999).

[48] Q.-F. Sun, J. Wang, and T. H. Lin, Phys. Rev. B 62, 648 (2000).

[49] J. C. Cuevas, A. Levy Yeyati, and A. Martín-Rodero, Phys. Rev. B 63, 094515 (2001).

[50] J. Baranski and T. Domanski, Phys. Rev. B 84, 195424 (2011).

[51] E. J. H. Lee, X. C. Jia, M. Houzet, R. Aguado, C. M. Lieber, and S. D. Franceschi, Nat. Nanotechnol. 9, 79 (2014).

[52] V. Kashcheyevs, A. Aharony, and O. Entin-Wohlman, Phys. Rev. B 73, 125338 (2006).

[53] Y. Qi, J. X. Zhu, and C. S. Ting, Phys. Rev. B 79, 205110 (2009).

[54] W. Chang, V. E. Manucharyan, T. S. Jespersen, J. Nygard, and C. M. Marcus, Phys. Rev. Lett. 110, 217005 (2013).

[55] R. Meservey, P. M. Tedrow, and P. Fulde, Phys. Rev. Lett. 25, 1270 (1970). 